\title{
Results on two-bit gate design for quantum computers
}

David P. DiVincenzo

IBM T. J. Watson Research Center

P. O. Box 218

Yorktown Heights, NY 10598

divince@watson.ibm.com
John Smolin 田

Dept. of Physics

University of California Los Angeles

Los Angeles, CA 90024

smolin@vesta.physics.ucla.edu

To be published in: Proceedings of the Workshop on Physics and Computation, PhysComp '94 (Los Alamitos: IEEE Comp. Soc. Press, 1994)

\begin{abstract}
We present numerical results which show how twobit logic gates can be used in the design of a quantum computer. We show that the Toffoli gate, which is a universal gate for all classical reversible computation, can be implemented using a particular sequence of exactly five two-bit gates. An arbitrary three-bit unitary gate, which can be used to build up any arbitrary quantum computation, can be implemented exactly with six two-bit gates. The ease of implementation of any particular quantum operation is dependent upon a very non-classical feature of the operation, its exact quantum phase factor.
\end{abstract}

\section{Introduction}

The quantum computer as a desirable future technology, which if achievable would revolutionize at least some areas of computational science, need hardly be promoted by the present contribution; the reader need only glance at some of the other papers presented at this conference 11, 22 to see the great promise (and perhaps overenthusiasm[3]) of this field.

A quantum computer may be contrasted with a classical computer in the following way: In a classical computer the elementary operations are boolean logic operations which are applied to a current state of the bits of the computer, resulting in a new bit state after a short operation time. These elementary logical operations are performed by logic gates, which typically operate on pairs of input bits, producing one output bit (e.g., XOR, AND, etc.). The quantum computer is very different: its elementary operations are unitary transformations applied to a wavefunction, that wavefunction being expressed as a complex linear superposition of the possible states of the computer, that is, of the possible states of its bits.

As in traditional computer design, it is necessary that the complete computation which is desired (i.e., the overall unitary transformation representing the complete computation) should be broken down into a discrete sequence of elementary logical operations. Thus, the "quantum logic gate" executes a unitary transformation which operates only on the part of the state of the system (i.e., the wavefunction) which involves a small subset of the bits of the computer. Such a "gate" involves a physical interaction among only the small subset of bits, and in this respect it is similar to a conventional logic gate. It differs from the classical logic gate in that the number of output bits must equal the number of input bits; quantum processes are reversible, and must involve no destruction of information.

Only a few useful quantum computations are known presently 1, 田, 包, but we can expect that more may be discovered in the future. Therefore, it is legitimate to ask the following general question: Given an arbitrary desired computation $U\left(2^{n}\right)$ (i.e., arbitrary unitary operation on $n$ bits), how may it be implemented by a sequence of quantum logic gates? Deutsch went most of the way towards answering this question in 1989[6], when he showed that just a single three-bit quantum logic gate is "universal", i.e., that a concatenation of this one gate applied in turn to different triplets of bits, could implement any $U\left(2^{n}\right)$. As an $8 \times 8$ matrix applied to the three-bit states $|0,0,0\rangle, \mid 0,0,1>, \ldots$ 
$\mid 1,1,1>$, the S-matrix of Deutsch's gate is:

$$
\mathbf{U}_{D}=\left(\begin{array}{cccccccc}
1 & & & & & & & \\
& 1 & & & & & & \\
& & 1 & & & & & \\
& & & 1 & 1 & & & \\
& & & & & 1 & & \\
& & & & & & i \cos \lambda & \sin \lambda \\
& & & & & & \sin \lambda & i \cos \lambda
\end{array}\right),
$$

where $\lambda$ is any constant such that $2 \lambda / \pi$ is an irrational number.

Deutsch's work left a few issues unsettled, however. He did not address the question of whether there might be a two-bit quantum logic gate which is universal. It was widely suspected that there was not, because it was known that no set of two-bit gates was sufficient for another formal system, classical reversible computation. Toffoli[] showed that for the classical problem, the minimal universal gate operates on three bits (this is now known as the Toffoli gate). Nevertheless, recently one of us showed that Deutsch's three-bit gates could be created by a sequence of two-bit quantum gates 8] (Deutsch's work had never excluded this possibility).

Deutsch's work also had nothing to say about the efficiency of the implementation of the $U\left(2^{n}\right)$ computation by quantum gates, and it is this issue which we will address in the present paper. Indeed, his calculation [6] only showed that an infinite sequence of $U\left(2^{3}\right)$ operations would be capable of approximating, to arbitrarily high accuracy, a desired $U\left(2^{n}\right)$; likewise, the recent proof of the universality of two-bit gates only shows that an infinite sequence of $U\left(2^{2}\right)$ operations would be capable of approximating a desired $U\left(2^{3}\right)$. A recent paper [9] shows how any $U(N)$ can be rewritten exactly as a finite sequence of simpler unitary operations; however, the elementary operations used in that paper do not operate on a small number of bits, and so do not qualify as "gates" in the present sense of the word. (See also [10] for a very closely related result.)

In this paper, then, we present a sequence of numerical experiments which explore the issue of efficient implementation. We show that an arbitrary $U\left(2^{3}\right)$ can indeed be expressed exactly using a sequence of (unconstrained) two-bit gates, and that the number of gates necessary for this exact implementation is six. We will also present the corresponding results for three-bit gates of special importance. We show that the Toffoli gate also requires exactly five two-bit gates for its exact implementation, which is somewhat surprising given its simple form. It has recently been shown 11] that Toffoli and Fredkin gates, but with the wrong quantum phases, can be exactly implemented in just three two-bit gates.

We will present a few other calculations illustrating more generally that the requirement of exact phase control 12 can substantially increase the complexity of implementation. Still, we view the bound of $6 \times$ on the complexity of implementation using two-bit operations as a hopeful one; it seems likely that it will often be desirable to pay this $6 \times$ complexity cost to be able to implement more physically realizable gates (see Conclusions). We will not in this paper address the larger implementation problem suggested by Deutsch's work, namely that of efficiently implementing the arbitrary $U\left(2^{n}\right)$ with a finite number of gates. Hopefully the present investigation will provide a basis on which to proceed on this harder problem.

\section{The Simulations}

We have attacked the problems listed above using "brute force" numerical experiments. While this approach generally does not permit any result to be rigorously proved, it does provide a quick way to generate a relatively large number of almost-certainly-correct results in an automatic way. While purely numerical, our approach takes advantage of a set of analytic results which could be more generally useful, so we mention them now.

\subsection{Notation and Constraints}

Figure 1illustrates the kinds of constructions which we have explored with the computer. It illustrates a particular analytic equivalence discovered in Ref. [8], between a particular three-bit gate designated " $U_{\lambda}$ " and a sequence of four two-bit gates (ignoring the two one-bit gates labeled "N" for the moment). Ref. [8] gives more details of the calculations which this diagram depicts. The horizontal lines in the figure may be taken to denote the world lines of the three bits (they may be spin- $1 / 2$ degrees of freedom in a physical implementation). The symbols are drawn to show that the first and third gates (the "X"'s) operate on bits 2 and 3 only, and the second and fourth gates ("V"'s) operate on bits 1 and 3 only.

In the numerical work, we explore an exhaustive set of two-bit gate topologies up to a certain maximum size. To describe our classification of these topologies, it is convenient to have a very compact notation for a particular arrangement of gates. We will use the following convention: the function of a two-bit gate will be denoted by the number of the bit on which it does 
not act. Obviously this is not a good notation for a general network, but suffices for the study of threebit networks which we conduct here. Thus, by this notation, the four-gate sequence in Fig. 1 is denoted (1212). Note that this designation is unchanged by the presence of the one-bit $\mathrm{N}$ gates; they can be absorbed into the $\mathrm{X}$ gates, with a corresponding redefinition of their operation. We will assume throughout this section that an arbitrary two-bit operation is permissible at every position. We will discuss briefly in the Conclusions the physical feasibility of this rule.

In the following computation it will be important to know all the possible distinct gate topologies, especially those with four or five two-bit gates. Two different topologies can be equivalent as networks for the following reasons:

Time-Reversal. When a sequence of unitary gates is operated in time-reversed order, the inverse unitary operation results. In all the cases which we consider below, the desired unitary operation is either selfinverse, or belongs to an ensemble which contains the inverse of every matrix. This means that the timereversed version of any topology is equally powerful in implementing the desired matrix or ensemble of matrices. So, for example, topologies (12123) and (32121) are equivalent, and we will write as shorthand $(12123)=(32121)$.

Bit Relabeling. The unitary matrices which we want to implement are in many cases invariant with respect to relabeling of the bits. If for example, the matrices are invariant under a relabeling of bits 1 and 2, then $(12123)=(21213)$.

Conjugation by Swapping. The swapping of the states of any pair of bits is a special two-bit logic gate[6], and this gate can be used to change the network topology. Figure 2 shows how this is done; the (3) gate in part a) can be changed to a (2) gate in part b) by the insertion of (1)-type swap gates, that is, those which interchange the states of bits 2 and 3 . This operation, the replacement $(3) \rightarrow(121)$, is a conjugation in the group-theoretic sense. It is generally not useful in identifying equivalent topologies of the same length, since it generally increases the number of gates. However, if a gate is surrounded by two gates operating on the same bits, then the swap gates can absorbed into the adjoining gates, as shown in Fig. 2(c). Thus, the general equivalence which conjugation implies is $(\mathrm{iji})=(\mathrm{iki})$, where $\mathrm{i}, \mathrm{j}$, and $\mathrm{k}$ are all different. For the five-gate network used as an example above, it produces the relations $(12123)=(13123)=(12323)=(12313)$.

Below we will use these three operations to produce irreducible sets of topologies to examine numerically.

\section{$2.2 \quad$ Numerical Technique}

To determine whether a particular three-bit operation can be implemented using a given network of two-bit gates, we employ a non-linear minimization procedure. The objective function for this minimization is constructed as follows: For each gate in the network, an $8 \times 8$ matrix is set up with a completely general parameterization of the gate. For example, if the two-bit gate is of type (3), then its S-matrix has the form

$$
S=\left(\begin{array}{cc}
U(4) & 0 \\
0 & U(4)
\end{array}\right)
$$

i.e., the matrix has a $4 \times 4$ block-diagonal form, with two identical copies of an arbitrary $U(4)$ matrix on the diagonal. The type (1) and (2) matrices are the same except for an appropriate re-ordering of the rows and columns. $S$ has $4^{2}=16$ free real-valued parameters 14.

Now, the unitary matrix of the complete network is given by a product of $\mathrm{S}$ matrices as in Eq. 2; call the resulting matrix $S^{t o t}$. If the number of gates in the network is $N_{2}, S^{\text {tot }}$ has $16 N_{2}$ free parameters (not necessarily all independent). If the desired three-bit operation has S-matrix $U$ (we will refer to this as the "target matrix" below), then we form the following objective function of $16 N_{2}$ variables:

$$
f=\sum_{i=1}^{8} \sum_{j=1}^{8}\left|U_{i j}-S_{i j}^{t o t}\right|^{2} .
$$

We then seek minima of $f$ numerically. If we find a minimum at which $f=0$ to reasonable numerical accuracy, then we have found the desired two-bit implementation of the three-bit gate. If all of the minima of the function have $f>0$, we conclude that the desired three-bit operation cannot be implemented by the two-bit topology being considered.

The numerical routine used was a variable-metric minimizer from the Harwell subroutine library, specifically the VF04AD routine. This routine introduces the unitarity of the individual matrices using Lagrange multipliers, and Broyden-Fletcher-GoldfarbShanno minimization is used [13]; the user is not required to supply expicitly a matrix of derivitives, which is helpful in dealing with complicated nonlinear functions such as those we have here. This routine is, of course, only capable of finding local minima, having no way to determine if the minimum is indeed global. 
Many such local minima are found in the present calculations, so the minimizer was in each case run starting from several sets of random initial conditions to ensure the real minimum could be found.

\subsection{Results: arbitrary $\mathrm{U}(8)$}

We have obtained convincing evidence that any $\mathrm{U}(8)$ target gate may be obtained by a network of six two-bit gates, and no fewer. We demonstrate this as follows: First, we find that using the six-gate topology (121212), a minimum of the objective function is obtained which is essentially zero, viz., $f_{\min } \leq 10^{-5}$. We have confirmed this with three different $\mathrm{U}(8)$ matrices generated randomly. (They are actually constructed from the eigenvectors of a random Hermitian matrix.) Second, we perform an exhaustive study of all distinct five-gate topologies. Using the symmetries mentioned above which the ensemble of all $\mathrm{U}(8)$ matrices possess-invariance under inversion, and under all relabelings of bits 1, 2, and 3-we need only consider two distinct five-gate topologies, (12121) and (12123). For neither of these topologies do we find a solution; in every case, the minimum value of the objective function $f$ is $f_{\text {min }} \geq 2.0$.

This shows, of course, that no topologies with fewer than 5 gates can implement the arbitrary $\mathrm{U}(8)$. It is worth noting, though, that there are simple analytic arguments that an arbitrary $\mathrm{U}(8)$ cannot be implemented in fewer than five gates. The number of parameters in the general $\mathrm{U}(8)$ is $8^{2}=64 \sqrt[14]{\text {. The num- }}$ ber of distinct parameters in the objective function for a network with $N_{2}$ gates is $16 N_{2}-\left(N_{2}-1\right)$. The second term in this expression comes from absorbing the overall phase factor of each two-bit gate into a single global phase factor (this amounts to considering SU(4) rather than $\mathrm{U}(4)$ matrices). Simple arithmetic shows that $N_{2}$ must be at least 5 in order that the objective function have at least 64 free parameters.

\subsection{Results: Toffoli gate}

In all known examples of quantum computing[1], 2, 4] it is essential that classical reversible logic gates be executed as quantum operations. All classical reversible logic can be generated by the Toffoli gate[7],

$$
\mathbf{U}_{T}=\left(\begin{array}{cccccccc}
1 & & & & & & & \\
& 1 & & & & & & \\
& & 1 & & & & & \\
& & & 1 & & & & \\
& & & & 1 & & & \\
& & & & & & 0 & 1 \\
& & & & & & 1 & 0
\end{array}\right),
$$

so we have explored the minimal implementation of this $\mathrm{U}(8)$ element. Of course, from the preceding section we know that it is implementable with six gates in the (121212) topology. We also find that the Toffoli gate can be achieved with five gates. We find that certain topologies work ((12123), (12132), (12312), (13213)) and others do not ((12121), (31213))[15. However, no simpler implementation of $U_{T}$ is possible, which we established by finding that the minimum of the objective function Eq. (3) was of order unity for the four-gate topologies (1231) and (3123). Thus, by the criterion of length of its two-gate implementation, the Toffoli gate is only slightly less complex that the general three-bit gate!

Previously, the best known implementation of the Toffoli gate was a six-gate implementation of topology (121231) found by Coppersmith [16]. (We do not obtain the same matrices as Coppersmith when we study this topology numerically, which is indicative of a weakness of our approach: since there are many unneeded free parameters in the (121231) implementation, our procedure does not pick out the one with "simple" two-bit gates which Coppersmith found.) Sleator and Weinfurter 17 have also recently shown a two-bit-gate implementation of $U_{T}$; while their implementation involves a greater number of gates, it respects the practical constraints involved in implementing unitary transformations in cavity QED, which of course have also not been considered here.

\subsection{Results: Margolus' modification of Toffoli-the phase problem}

Our results demonstrate that the ease of implementation of the quantum gate depends very markedly upon its phases, a situation which has no classical analog. To illustrate this, we quote a result from N. Margolus 11] for the implementation of a slight modification of the Toffoli gate:

$$
\mathbf{U}_{M}=\left(\begin{array}{cccccccc}
1 & & & & & & & \\
& 1 & & & & & & \\
& & 1 & & & & & \\
& & & 1 & & & & \\
& & & & 1 & -1 & & \\
& & & & & & 0 & 1 \\
& & & & & & 1 & 0
\end{array}\right),
$$

which differs from $U_{T}$ only in that it changes the phase of the $|1,0,1\rangle$ component of the quantum state. Despite this minor change, Margolus has shown that $U_{T}$ can be implemented with just three two-bit gates, in the (121) topology. (By checking (12), (13) and (23), we have shown that Margolus' result is minimal. We 
also find that for the (121) topology, other choices of phase factors in the matrix Eq. (5) besides Margolus' can be implemented.)

To further illustrate the difficulty of achieving the desired phase in quantum gates, we investigated the two-bit implementation of perhaps the simplest possible three-bit gate, which Deutsch[6] calls "X":

$$
\mathbf{U}_{\phi}=\left(\begin{array}{cccccccc}
1 & & & & & & & \\
& 1 & & & & & & \\
& & 1 & & & & & \\
& & & 1 & & & & \\
& & & & 1 & & & \\
& & & & & & 1 & \\
& & & & & & & e^{i \phi}
\end{array}\right) \text {, }
$$

for which the state is unchanged except for the modification of one phase factor. It turns out that even this simple matrix is not very simple to implement with two-bit gates. It can of course be constructed in the "universal" 6-gate topology (121212); the 5gate topologies (12123) and (12312) work, although (12121) fails; and, no 4-gate topology can implement $U_{\phi}$, as we established by checking (1231) ((1212) is already excluded by the 5 -gate result). So, its complexity is the same as $U_{T}$ 's. As Denker has recently emphasized 12], having incorrect phases in a quantum computation could be catastrophic, e.g., for a quantum Fourier transform operation 11, 5]; thus, $U_{M}$ can be used in place of $U_{T}$ only with great care, and "simple" phase adjustments like $U_{\phi}$ must be carefully designed. The present results show that these seemingly minor adjustments may exact a considerable cost in network complexity.

\section{Concluding Remarks}

There are several obvious weaknesses to the present results, which we would like to touch on. First, since the approach is purely numerical, no result in this paper can be taken as strictly proved, only strongly suggested. In [8] we proved, using the generator calculus of the Lie groups, that representation of any threebit gate by a sequence of two-bit gates was possible, provided that arbitrarily many two-bit gates are permitted. To our disappointment, we have found no way of adapting this calculus to prove the stronger results, that operations are implementable exactly in a finite number of gates. Hopefully by producing a set of definite results here, we may be led to some new way of applying Lie group theory to obtain the desired stronger analytic results.

A second weakness of the present approach is a more physical one: we assume that any arbitrary two-bit unitary operation is possible (see Eq. (2)). Whether this is true or not depends on the details of the physical implementation of the quantum system. As Ref. [17] illustrates, for any specific system (e.g., cavity QED) certain two-bit operations are much easier to implement that others. It seems likely that in most circumstances where several different two-bit operations are easy to accomplish, that suitable concatenations of these operations can generate any arbitrary $U(4)$; probably the most is known about this in the context of multiple-resonance NMR 18]. Still, no definite results have been obtained on this question yet, and it should be the subject of future study. It would also be sensible to include the ease of construction of particular classes of $U(4)$ operations into our numerical optimizations.

To summarize: Our numerical studies indicate that any arbitrary three-bit quantum gate can be produced exactly as a concatenation of six two-bit quantum gates. A particular three-bit logic gate of great importance, the Toffoli gate, can be produced with five. Relaxing the constraint that the Toffoli gate introduce no phase shifts, which is often a dangerous thing to do, leads to a three-gate implementation with two-bit operations, as shown by Margolus. The insertion of even the simplest additional phase shift in the threebit space requires five additional two-bit gates.

\section{Acknowledgements}

We are grateful to C. H. Bennett, H. J. Bernstein and R. Landauer for many helpful discussions.

\section{References}

[*] Also at IBM Thomas J. Watson Research Center.

[1] P. W. Shor, "Algorithms for quantum computation: discrete log and factoring", these proceedings; see also Proceedings of the 35th IEEE Symposium on the Foundations of Computer Science, 1994 (to be published).

[2] A. Berthiaume, D. Deutsch, and R. Jozsa, "The stabilisation of quantum computations", these proceedings.

[3] R. Landauer, "Zig-zag path to understanding", these proceedings; see also R. Landauer, "Is quantum mechanics useful?", Proc. Roy. Soc. Lond., (to be published).

[4] W. K. Wootters, "Quantum block coding", unpublished (1994). 
[5] D. Coppersmith, "An approximate Fourier transform useful in quantum factoring", IBM Research Report RC19642 (1994).

[6] D. Deutsch, "Quantum computational networks", Proc. Roy. Soc. Lond. A 425, 73 (1989).

[7] T. Toffoli "Reversible Computing", in Automata, Languages and Programming, eds. J. W. de Bakker and J. van Leeuwen (Springer, New York, 1980), p. 632; Technical Memo MIT/LCS/TM151, MIT Lab. for Comp. Sci. (unpublished).

[8] D. P. DiVincenzo, "Two-bit gates are universal for quantum computation", submitted to Phys. Rev. A (1994).

[9] M. Reck, A. Zeilinger, H. J. Bernstein, and P. Bertani, "Experimental realization of any discrete unitary operator", Phys. Rev. Lett. 73, 58 (1994).

[10] A. Ekert and R. Jozsa, "Notes on Shor's efficient algorithm for factoring on a quantum computer", Workshop on Quantum Computing and Communication, Gaithersburg, MD, August 18-19, 1994, to appear on WWW.

[11] N. Margolus, private communication.

[12] A point recently anticipated by J. S. Denker, Workshop on Quantum Computing and Communication, Gaithersburg, MD, August 18-19, 1994 (unpublished).

[13] W. H. Press, B. P. Flannery, S. A. Teukolsky, W. T. Vetterling, Numerical Recipes (Cambridge University Press, 1986), Chap. 10.

[14] J. Mathews and R. L. Walker, Mathematical Methods of Physics, (Benjamin, Second Edition, 1970), Chap. 16, has a basic review of the theory of unitary matrices.

[15] These topologies are all the distinct ones, taking account of the fact that the Toffoli gate is not invariant under any bit relabeling involving bit 3 .

[16] D. Coppersmith, unpublished (1994).

[17] T. Sleator and H. Weinfurter, "Quantum teleportation and quantum computation based on cavity QED", Ann. NY Acad. Sci., to be published. (Presented at the Conference on Fundamental Problems in Quantum Theory, June 18-22, 1994, Baltimore, MD.)
[18] C. P. Slichter, Principles of Magnetic Resonance (Third Edition, Springer-Verlag, 1992). 

(a)

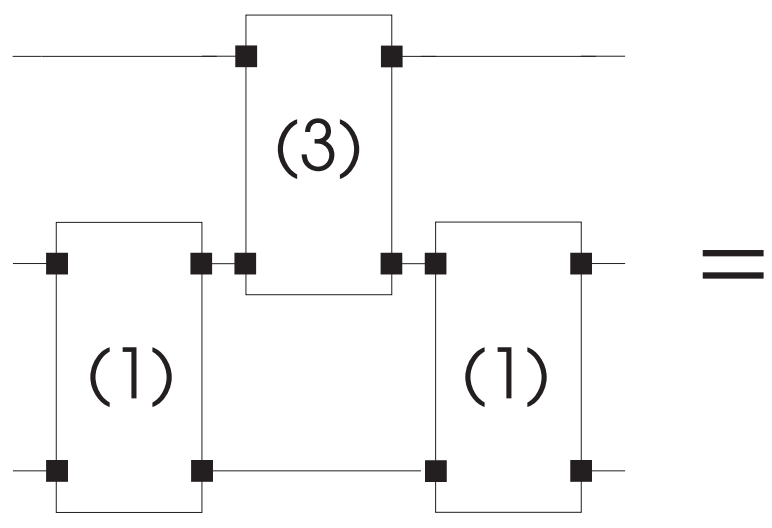

(b)

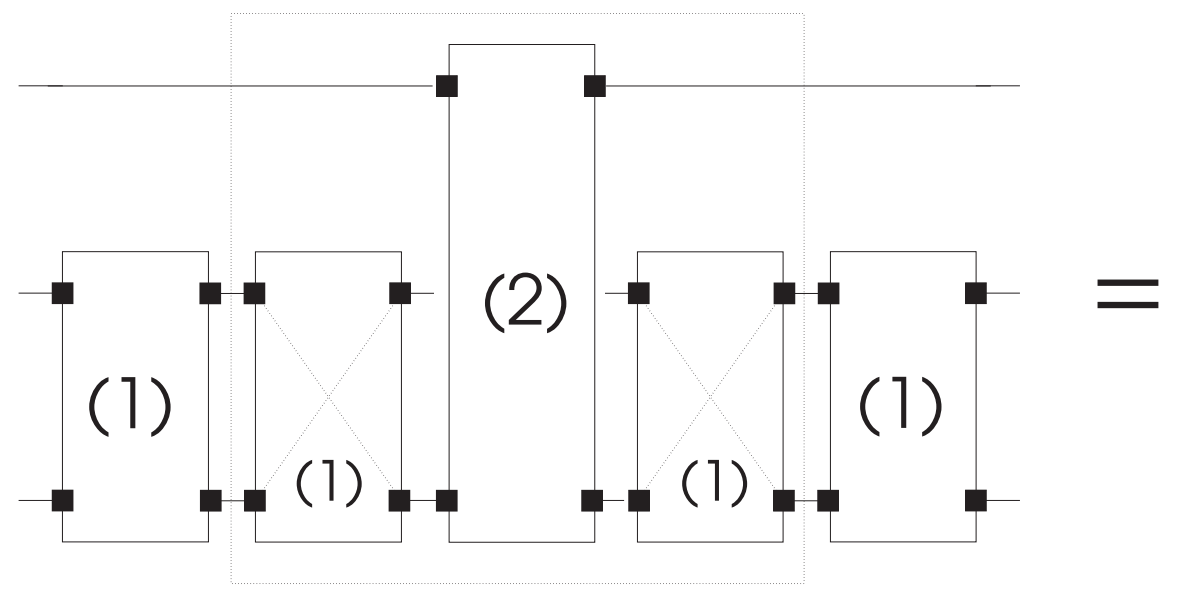

(c)

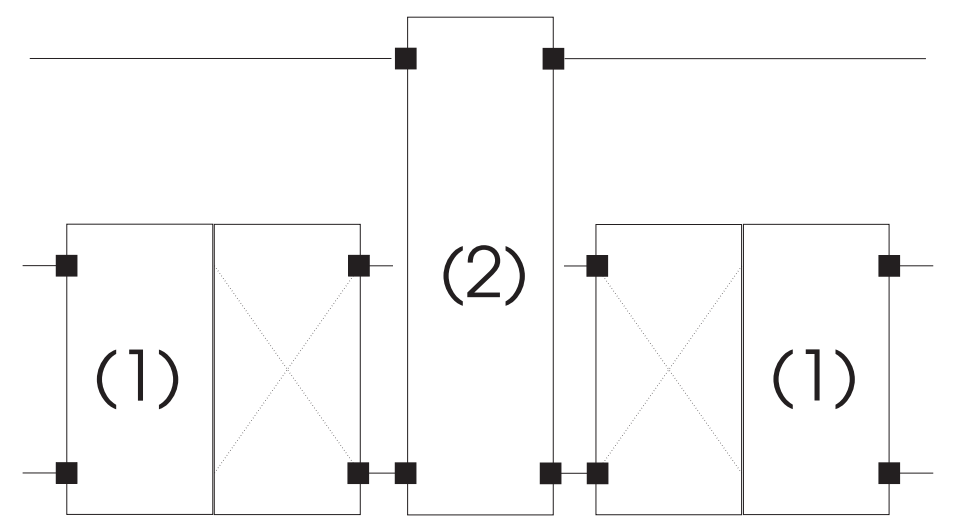

Figure 1: Illustration of a replacement of a three-bit gate by a sequence of one- and two-bit gates. The numbering convention $(1,2,3)$ for three bits is indicated. The one bit "N" gate may be absorbed into the definition of the two-bit gates. According to the notation discussed in the text, the network shown has the topology (1212). For more details of the use of this particular decomposition, see [8]. 

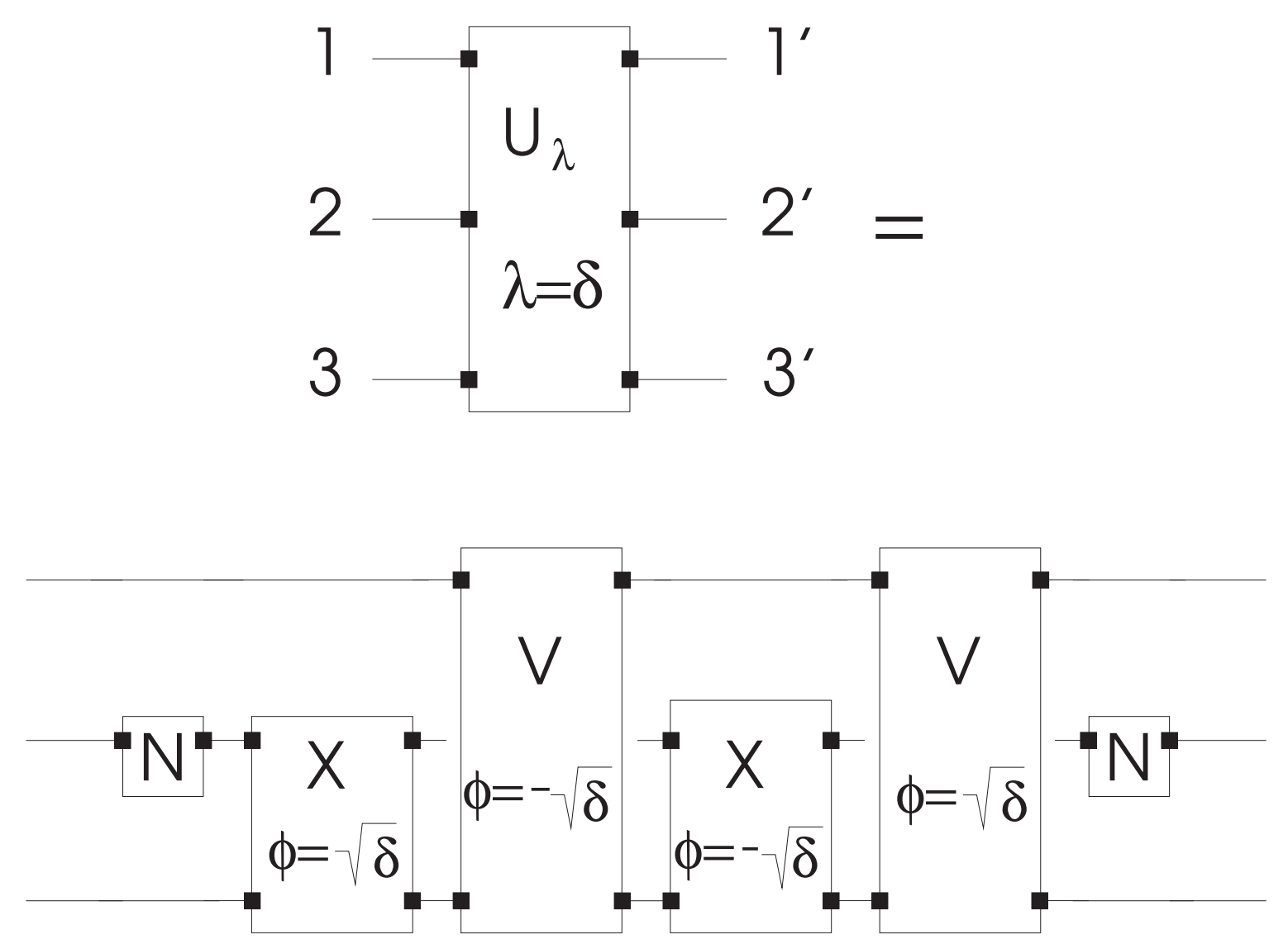

Figure 2: Modification of network topology by swapping. The (131) topology of (a) is changed in (b) by replacing (3) by the set of gates in the dotted box: a (2) and two surrounding swapping gates of the (1) type. The pairs of (1)'s are then merged together in (c). The net result is the replacement $(131) \rightarrow(121)$. 